\title{
IRF4 expression is low in Philadelphia negative myeloproliferative neoplasms and is associated with a worse prognosis
}

\author{
Cosimo Cumbo ${ }^{1 \dagger}$, Francesco Tarantini ${ }^{1 \dagger}$, Luisa Anelli ${ }^{1}$, Antonella Zagaria', Immacolata Redavid ${ }^{1}$, \\ Crescenzio Francesco Minervini ${ }^{1}$, Nicoletta Coccaro', Giuseppina Tota' , Alessandra Ricco', Elisa Parciante', \\ Maria Rosa Conserva', Giorgina Specchia ${ }^{2}$, Pellegrino Musto ${ }^{1}$ and Francesco Albano ${ }^{1 *}$ (D)
}

\begin{abstract}
Interferon regulatory factor 4 (IRF4) is involved in the pathogenesis of various hematologic malignancies. Its expression has been related to the negative regulation of myeloid-derived suppressor cells (MDSCs) and the polarization of anti-inflammatory M2 macrophages, thereby altering immunosurveillance and inflammatory mechanisms. An abnormal inflammatory status in the bone marrow microenvironment of myeloproliferative neoplasms (MPNs) has recently been demonstrated; moreover, in chronic myeloid leukemia a downregulated expression of IRF4 has been found. In this context, we evaluated the IRF4 expression in 119 newly diagnosed consecutive Philadelphia negative MPNs (PhMPNs), showing a low expression among the MPNs phenotypes with a more significant decrease in primary myelofibrosis patients. Lower IRF4 levels were associated with JAK2 + and triple negatives cases carrying the worst prognosis. Furthermore, the IRF4 levels were related to leukemic transformation and a shorter leukemia-free survival; moreover, the risk of myelofibrosis transformation in polycythemia vera and essential thrombocythemia patients was more frequent in cases with lower IRF4 levels. Overall, our study demonstrates an IRF4 dysregulated expression in MPNs patients and its association with a worse prognosis. Further studies could validate these data, to improve our knowledge of the MPNs pathogenesis and confirm the IRF4 role as a new prognostic factor.
\end{abstract}

Keywords: IRF4 expression, Philadelphia negative MPNs, Prognosis

\section{To the Editor}

Interferon regulatory factor 4 (IRF4) is a transcription factor with an established role in the pathogenesis of various hematologic malignancies [1]. IRF4 expression has been related to the negative regulation of myeloid-derived suppressor cells (MDSCs), thereby altering immunosurveillance; moreover, its expression drives inflammation through the polarization of

\footnotetext{
*Correspondence: francesco.albano@uniba.it

${ }^{\dagger}$ Cosimo Cumbo and Francesco Tarantini contributed equally to this work ${ }^{1}$ Department of Emergency and Organ Transplantation (D.E.T.O.),

Hematology and Stem Cell Transplantation Unit, University of Bari "Aldo Moro", P.Zza G. Cesare, 11, 70124 Bari, Italy

Full list of author information is available at the end of the article
}

anti-inflammatory M2 macrophages [2, 3]. In chronic myeloid leukemia a downregulated expression of IRF4 has been found [4] and recent evidence supports its prognostic role in JAK2V617F mutated myeloproliferative neoplasms (MPNs) [5].

With these premises, we evaluated IRF4 expression in 119 Philadelphia negative MPNs (Ph- MPNs) patients (Additional file 2: Table S1) to verify its role on clinical outcome (median follow-up: 61.5 months, range: 1-238). The quantification was calculated as the ratio between IRF4 and GUSB number of copies (I/G) (Additional file 1).

The bone marrow (BM) IRF4 median value was 0.11 I/G (min. 0.11- max. 012) and 0.04 I/G (min. 0.001 
- max. 0.27) in the healthy and in the MPNs groups, respectively $(\mathrm{p}<0.0001)$. Considering the IRF4 median value for every MPN type, the difference compared to the healthy controls $(\mathrm{HC})$ remained statistically significant (Fig. 1A). In particular, the primary myelofibrosis (PMF) patients showed a lower IRF4 median value than that of the other groups compared to the HC. Among the MPNs, the PMF IRF4 median value was lower than in the essential thrombocythemia (ET) group $(\mathrm{p}=0.003)$. CALR mutated cases showed a higher IRF4 expression than those with JAK2 (0.06 vs $0.03, \mathrm{p}=0.007)$ or triple-negative $(\mathrm{TN})(0.06 \mathrm{I} / \mathrm{G}$ vs $0.02 \mathrm{I} / \mathrm{G}, \mathrm{p}=0.0008$ ) (Fig. 1B). IRF4 expression was not associated with variables as sex, age, risk group [6]. Fourteen (11.7\%) patients showed leukemic transformation (LT): 8 PMF, 4 secondary myelofibrosis (SMF), and 2 polycythemia vera (PV); they had a lower IRF4 expression at diagnosis compared to the other MPN patients $(0.01 \mathrm{I} / \mathrm{G}$ vs $0.04 \mathrm{I} / \mathrm{G}, \mathrm{p}=0.0005)$ (Fig. 1C). An optimal cutoff of the IRF4 expression value best identifying the possibility of MPN leukemic transformation was defined by ROC analysis. The area under the curve was 0.79 (95\% CI 0.71-0.86; p < 0.0001). Representative cutoff values for sensitivity and specificity were calculated and plotted on the curve. An optimal value of $0.022 \mathrm{I} / \mathrm{G}$ was obtained, with a sensitivity of $76.9 \%$ (95\% CI $46.2-95.0)$ and a specificity of $76.1 \%$ (95\% CI $66.9-$ 84.0). This value distinguished MPN patients with a higher probability of LT; in fact, the group with an IRF4 value $<0.022 \mathrm{I} / \mathrm{G}$ had shorter leukemia-free survival
(LFS) (Fig. 2A). The LFS analysis was also considered only for the myelofibrosis (MF) group; patients with an IRF4 value $<0.022 \mathrm{I} / \mathrm{G}$ showed a shorter LFS $(\mathrm{p}=0.001$, Fig. 2B). In another LFS analysis, this difference was confirmed when considering only the PMF group: the median LFS for PMF patients with IRF4 $<0.022 \mathrm{I} / \mathrm{G}$ was 23 months, whereas LFS was not reached for the $>0.022$ $\mathrm{I} / \mathrm{G}$ group $(\mathrm{p}=0.0009)$ (Fig. $2 \mathrm{C})$. Overall survival $(\mathrm{OS})$ analysis in the MF group showed that patients with IRF4 $>0.002 \mathrm{I} / \mathrm{G}$ had longer median survival than those in the $<0.022 \mathrm{I} / \mathrm{G}$ group (143.9 mo. versus $40.5 \mathrm{mo}$., $\mathrm{p}=0.04$ ) (Fig. 2D). Also, in the PMF group, the IRF4 value $>0.022 \mathrm{I} / \mathrm{G}$ was associated with a longer OS (75 mo versus $21 \mathrm{mo}, \mathrm{p}=0.04$ ) (Fig. $2 \mathrm{E}$ ). Moreover, $\mathrm{PV}$ and ET patients with an IRF4 value $<0.022$ I/G showed a shorter time to MF transformation (109 mo. versus 190 mo., $\mathrm{p}=0.03$ ) (Fig. 2F).

Among the 14 patients with LT, 9 (64.2\%), 7 PMF and 2 SMF, were analyzed in next-generation sequencing [7] to detect the high molecular risk (HMR) mutations (Additional file 3: Table S2). In 5 PMF patients, HMR mutations were found; moreover, all 9 patients showed at least one additional genetic lesion besides the gene driver mutation (Additional file 4: Figure S1). Despite the data paucity, just under half of the patients with MF who had undergone LT (44.4\%) did not have HRM mutations. In three PMF cases, the IRF4 expression was evaluated during ruxolitinib treatment. All cases exhibited an increased IRF4 expression compared to the value at diagnosis $(\mathrm{p}=0.003)$; in the two patients with a longer

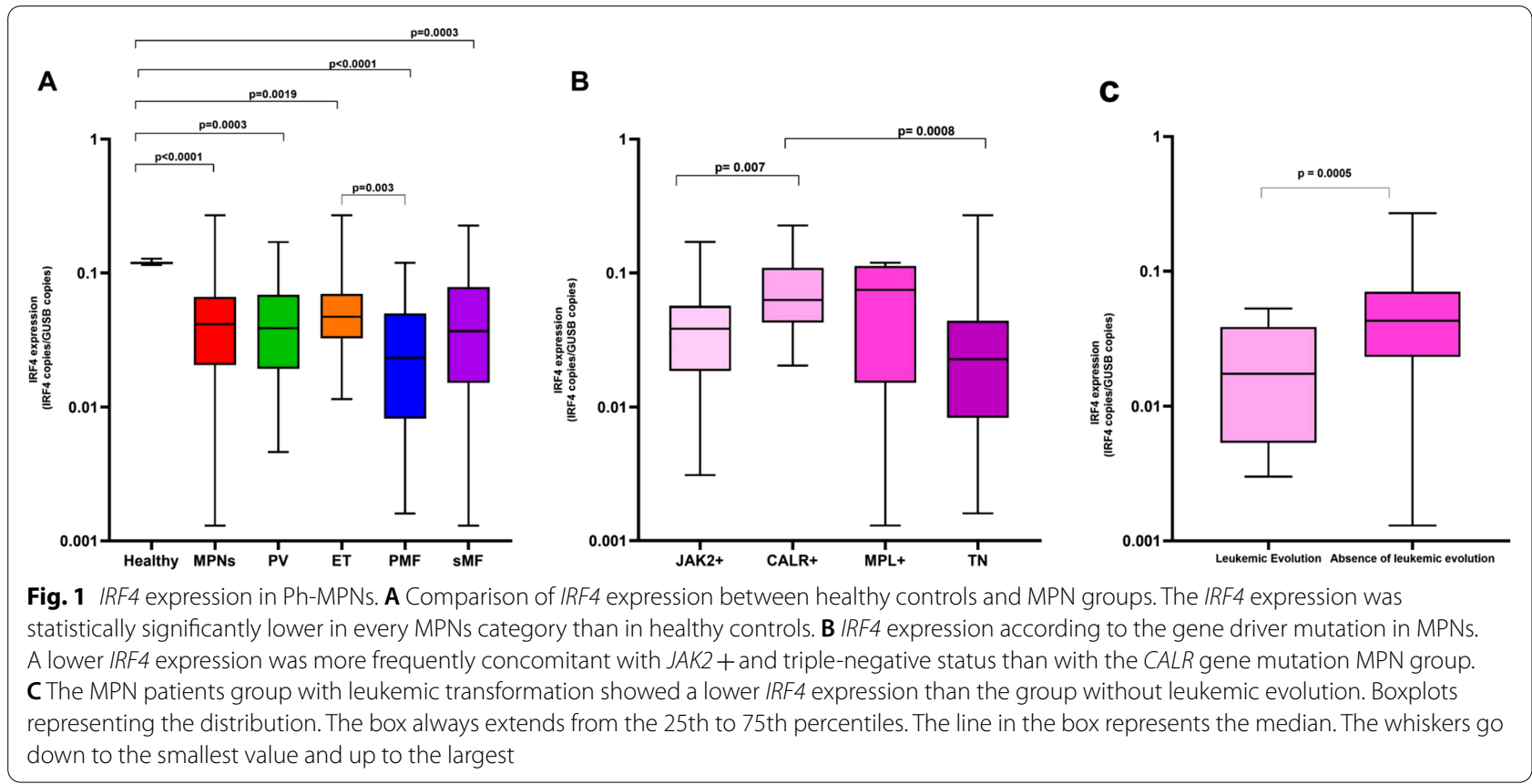




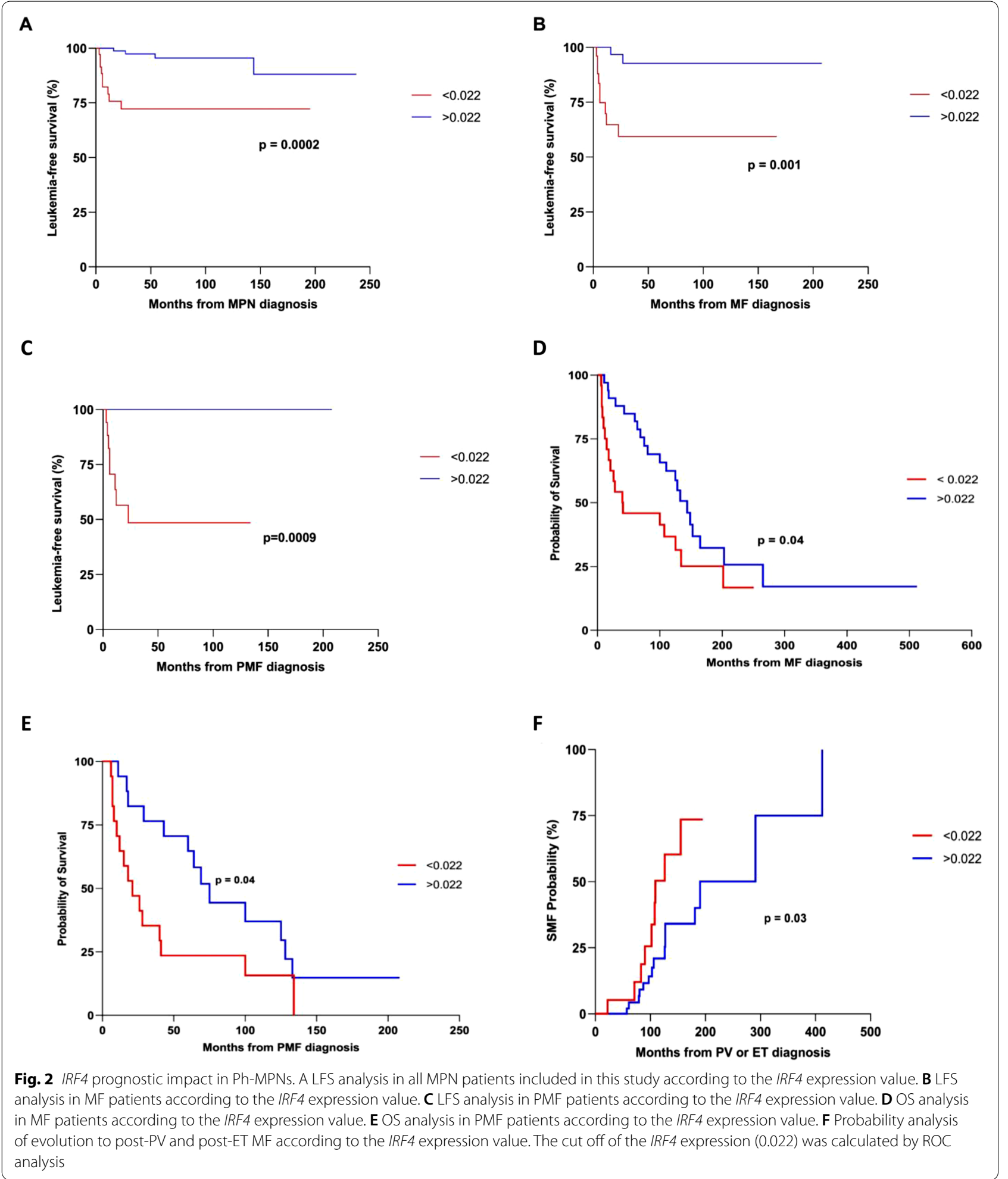

duration of ruxolitinib treatment $(59$ mo. and 72 mo., respectively), there was a one-log increment of IRF4 expression (Additional file 5: Figure S2).
Despite the main limits of our study (relatively small number of patients in each group, few NGS data, inability to determine the IRF4 production source) 
we demonstrate an IRF4 dysregulated expression in MPNs patients, particularly in PMF and in $J A K 2+$ and $\mathrm{TN}+$ cases, distinguishing those with a higher probability of SMF. Furthermore, the IRF4 expression was associated with LT and a shorter LFS. Further studies are warranted to validate these data to confirm this biomarker as a new prognostic factor.

\begin{abstract}
Abbreviations
IRF4: Interferon regulatory factor 4; MDSCs: Myeloid-derived suppressor cells; MPNs: Myeloproliferative neoplasms; Ph-MPNs: Philadelphia negative myeloproliferative neoplasms; BM: Bone marrow; HC: Healthy controls; PMF: Primary myelofibrosis; ET: Essential thrombocythemia; TN: Triple negative; LT: Leukemic transformation; SMF: Secondary myelofibrosis; PV: Polycythemia vera; LFS: Leukemia-free survival; MF: Myelofibrosis; OS: Overall survival; HMR: High molecular risk.
\end{abstract}

\section{Supplementary Information}

The online version contains supplementary material available at https://doi. org/10.1186/s40164-021-00253-y.

Additional file 1: Supplementary methods. Detailed experimental procedures for droplet digital PCR, NGS and statistical analysis.

Additional file 2: Table S1. Main patients' data. MPN patients biological and clinical characteristics.

Additional file 3: Table S2. NGS results. VCF file reporting the annotation of variants identified by NGS analysis.

Additional file 4: Figure S1. Oncoprinter visualization of NGS results. Variants identified for all cases analyzed (columns) are reported. The percentage value associated with each gene indicates its variants occurring in the cohort analyzed.

Additional file 5: Figure S2. Expression analysis of the IRF4 gene transcript at diagnosis and during ruxolitinib treatment in three myelofibrosis patients. The amount of IRF4 gene transcript was significantly increased during ruxolitinib therapy in all cases analyzed.

\section{Acknowledgements}

This work was supported by "Associazione Italiana contro le Leucemie (AIL)-BARI".

\section{Authors' contributions}

Conception and design of the study: CC, FT and FA. Acquisition of data and/ or analysis and interpretation of data: CC, FT, LA, AZ, IR, CFM, NC, GT, AR, EP, MRC, GS, PM and FA. Drafting of the manuscript: FA. All authors revised the manuscript for important intellectual content and approved the final version submitted for publication. All authors read and approved the final manuscript.

\section{Funding}

Not applicable.

\section{Availability of data and materials}

Not applicable.

\section{Declarations}

\section{Ethics approval and consent to participate}

The local ethics committee approved the study. Informed consent was obtained from all patients before study inclusion, in accordance with the Declaration of Helsinki. Patients' records/information were anonymized and de-identified before analysis.

\section{Consent for publication}

Consent for publication was obtained from patients before their enrolment in the present study.

\section{Competing interests}

The authors declare that they have no competing interests.

\section{Author details}

${ }^{1}$ Department of Emergency and Organ Transplantation (D.E.T.O.), Hematology and Stem Cell Transplantation Unit, University of Bari "Aldo Moro", P.Zza G. Cesare, 11, 70124 Bari, Italy. ${ }^{2}$ School of Medicine, University of Bari "Aldo Moro", 70124 Bari, Italy.

Received: 4 November 2021 Accepted: 18 December 2021

Published online: 24 December 2021

\section{References}

1. Yanai $\mathrm{H}$, Negishi $\mathrm{H}$, Taniguchi $T$. The IRF family of transcription factors inception, impact and implications in oncogenesis. Oncoimmunology. 2012;1:1376-86.

2. Longhitano L, Li Volti G, Giallongo C, Spampinato M, Barbagallo I, Di Rosa $M$, et al. The role of inflammation and inflammasome in myeloproliferative disease. J Clin Med. 2020;9:2334.

3. Satoh T, Takeuchi O, Vandenbon A, Yasuda K, Tanaka Y, Kumagai Y, et al. The Jmjd3-Irf4 axis regulates M2 macrophage polarization and host responses against helminth infection. Nat Immunol. 2010;11:936-44.

4. Schmidt M, Hochhaus A, König-Merediz SA, Brendel C, Proba J, Hoppe GJ, et al. Expression of interferon regulatory factor 4 in chronic myeloid leukemia: correlation with response to interferon alfa therapy. J Clin Oncol. 2000;18:3331-8.

5. Huang $X, M a T$, Zhu Y, Jiao B, Yu S, Wang $K$, et al. IRF4 and IRF8 expression are associated with clinical phenotype and clinico-hematological response to hydroxyurea in essential thrombocythemia. Front Med. 2021. https://doi.org/10.1007/s11684-021-0858-1.

6. Cervantes F, Dupriez B, Pereira A, Passamonti F, Reilly JT, Morra E, et al. New prognostic scoring system for primary myelofibrosis based on a study of the International working group for myelofibrosis research and treatment. Blood. 2009;113:2895-901. https://doi.org/10.1182/ blood-2008-07-170449.

7. Coccaro N, Zagaria A, Orsini P, Anelli L, Tota G, Casieri P, et al. RARA and RARG gene downregulation associated with EZH2 mutation in acute promyelocytic-like morphology leukemia. Hum Pathol. 2018;80:82-6.

\section{Publisher's Note}

Springer Nature remains neutral with regard to jurisdictional claims in published maps and institutional affiliations.

Ready to submit your research? Choose BMC and benefit from

- fast, convenient online submission

- thorough peer review by experienced researchers in your field

- rapid publication on acceptance

- support for research data, including large and complex data types

- gold Open Access which fosters wider collaboration and increased citations

- maximum visibility for your research: over 100M website views per year

At $\mathrm{BMC}$, research is always in progress.

Learn more biomedcentral.com/submissions 